\title{
Convergence of the all-time supremum of a Lévy process in the heavy-traffic regime
}

\author{
K.M. Kosiński • O.J. Boxma • B. Zwart
}

Received: 1 July 2010 / Revised: 9 December 2010 / Published online: 10 March 2011

(C) The Author(s) 2011. This article is published with open access at Springerlink.com

\begin{abstract}
In this paper we derive a technique for obtaining limit theorems for suprema of Lévy processes from their random walk counterparts. For each $a>0$, let $\left\{Y_{n}^{(a)}: n \geq 1\right\}$ be a sequence of independent and identically distributed random variables and $\left\{X_{t}^{(a)}: t \geq 0\right\}$ be a Lévy process such that $X_{1}^{(a)} \stackrel{d}{=} Y_{1}^{(a)}, \mathbb{E} X_{1}^{(a)}<0$ and $\mathbb{E} X_{1}^{(a)} \uparrow 0$ as $a \downarrow 0$. Let $S_{n}^{(a)}=\sum_{k=1}^{n} Y_{k}^{(a)}$. Then, under some mild assumptions, $\Delta(a) \max _{n \geq 0} S_{n}^{(a)} \stackrel{d}{\rightarrow} \mathcal{R} \Longleftrightarrow \Delta(a) \sup _{t \geq 0} X_{t}^{(a)} \stackrel{d}{\rightarrow} \mathcal{R}$, for some random variable $\mathcal{R}$ and some function $\Delta(\cdot)$. We utilize this result to present a number of limit theorems for suprema of Lévy processes in the heavy-traffic regime.
\end{abstract}

Keywords Lévy processes · Heavy traffic · Functional limit theorems · Mittag-Leffler distribution

The first author was supported by NWO Grant 613.000.701.

K.M. Kosiński (凶) · O.J. Boxma

Eurandom, Eindhoven University of Technology, P.O. Box 513, 5600 MB Eindhoven,

The Netherlands

e-mail: Kosinski@eurandom.tue.nl

K.M. Kosiński

Korteweg-de Vries Institute for Mathematics, University of Amsterdam, P.O. Box 94248, 1090 GE Amsterdam, The Netherlands

O.J. Boxma

Department of Mathematics and Computer Science, Eindhoven University of Technology, P.O. Box 513, 5600 MB Eindhoven, The Netherlands

e-mail: Boxma@win.tue.nl

B. Zwart

CWI, P.O. Box 94079, 1090 GB Amsterdam, The Netherlands

e-mail: Bert.Zwart@cwi.nl 
Mathematics Subject Classification (2000) Primary 60G50 • 60G51 · Secondary $60 \mathrm{~K} 25 \cdot 60 \mathrm{~F} 17$

\section{Introduction}

For each $a>0$, let $X^{(a)} \equiv\left\{X_{t}^{(a)}: t \geq 0\right\}$ be a Lévy process such that $\mu^{(a)}:=$ $\mathbb{E} X_{1}^{(a)}<0$. Along with the Lévy process $X^{(a)}$ define $\bar{X}^{(a)}=\sup _{t \geq 0} X_{t}^{(a)}$. Since $\mu^{(a)}<0$ assures that $X^{(a)}$ drifts to $-\infty$, the all-time supremum $\bar{X}^{(a)}$ is a proper random variable for each $a>0$. Now if $\mu^{(a)} \uparrow 0$ as $a \downarrow 0$, then $\bar{X}^{(a)} \rightarrow \infty$. From this fact a natural question arises: How fast does $\bar{X}^{(a)}$ grow as $a \downarrow 0$ ?

The main purpose of this paper is to answer the above question by considering the discrete approximation of a Lévy process by a random walk. For each $a>0$, let $\left\{Y_{n}^{(a)}: n \geq 1\right\}$ be a sequence of independent and identically distributed (i.i.d.) random variables. Put $\bar{S}^{(a)}=\max _{n \geq 0} S_{n}^{(a)}$, where $S_{n}^{(a)}=\sum_{k=1}^{n} Y_{k}^{(a)}, S_{0}^{(a)}=0$. We shall show that if $Y_{1}^{(a)}$ has the same distribution as $X_{1}^{(a)}$, then the limiting distribution of $\bar{X}^{(a)}$ can be derived from the limiting distribution of $\bar{S}^{(a)}$. In doing so we shall utilize a bound by Willekens [25]. Loosely speaking, this bound allows us to derive certain properties of Lévy processes via their corresponding random walk approximations (see also Doney [9]). The advantage of this approach, that is, changing from $\bar{X}^{(a)}$ to $\bar{S}^{(a)}$, is that the problem on how fast $\bar{S}^{(a)}$ grows as $a \downarrow 0$ has been treated extensively and various methods have been developed.

One major reason why the behavior of $\bar{S}^{(a)}$ has been studied is that it is well known that the stationary distribution of the waiting time of a customer in a singleserver first-come-first-served $G I / G I / 1$ queue coincides with the distribution of the maximum of a corresponding random walk. The condition for the mean of the random walk to become small ( $a \downarrow 0$ ) means in the context of a queue that the traffic load tends to 1 . Thus, the problem under consideration (in the random walk setting) may be seen as the investigation of the growth rate of the stationary waiting-time distribution in a $G I / G I / 1$ queue. This is one of the most important problems in queueing theory that is referred to as the heavy-traffic approximation problem. The question was first posed by Kingman (see [15] for an extensive discussion on the early results). It has been solved in various settings by, e.g., Prokhorov [18], Boxma and Cohen [5], Resnick and Samorodnitsky [20], Szczotka and Woyczyński [24] and many others.

Surprisingly, there are no results in the literature on the heavy-traffic limit theorems for Lévy-driven (fluid) queues. Our approach however allows us to translate each single result in the random walk setting to its analogue in the Lévy setting, thereby providing a range of fluid heavy-traffic limit theorems. Our main result, Theorem 1 , states: under some mild conditions, $\Delta(a) \bar{S}^{(a)} \stackrel{d}{\rightarrow} \mathcal{R}$ if and only if $\Delta(a) \bar{X}^{(a)} \stackrel{d}{\rightarrow} \mathcal{R}$, for some random variable $\mathcal{R}$ and some function $\Delta(\cdot)$.

The remainder of the paper is organized as follows. In Sect. 2 we fix notation and give some necessary preliminaries. Section 3 contains the main result of this paper, Theorem 1, and its proof. Instances of this theorem applied to the results by Boxma and Cohen [5], Shneer and Wachtel [21] and Szczotka and Woyczyński [23] (see also Czystołowski and Szczotka [8]) are presented in Sect. 4 and conclude the paper. 


\section{Preliminaries and notation}

All the stochastic objects are assumed to be defined on the probability space $(\Omega, \mathbb{F}, \mathbb{P})$ endowed with a standard filtration $\mathcal{F}=\left\{\mathcal{F}_{t}: t \geq 0\right\}$; that is $\mathcal{F}$ is an increasing, rightcontinuous family of complete sub- $\sigma$-fields of $\mathbb{F}$. Let us begin by fixing the notation for Lévy processes.

A real-valued stochastic process $X \equiv\left\{X_{t}: t \geq 0\right\}$, with $X_{0}=0$, is said to be a Lévy process with respect to the filtration $\mathcal{F}$ if it is adapted to $\mathcal{F}, X_{s}-X_{t}$ is independent of $\mathcal{F}_{t}$ and distributed as $X_{s-t}$ for any $0 \leq t<s$. Moreover, we assume that the sample paths of $X$ are càdlàg (right-continuous with left limits), so that $X$ is strong Markov.

Let $\psi$ be the Lévy characteristic exponent of $X$ so that $\mathbb{E} e^{i u X_{t}}=e^{-t \psi(u)}$, for all $u \in \mathbb{R}$. In this case, for some $\sigma>0$ and $\delta \in \mathbb{R}, \psi$ has the form

$$
\psi(u)=i \delta u+\frac{1}{2} \sigma^{2} u^{2}+\int_{|x|<1}\left(1-e^{i u x}+i u x\right) v(d x)+\int_{|x| \geq 1}\left(1-e^{i u x}\right) v(d x),
$$

where $v$ is the Lévy measure (on $\mathbb{R} \backslash\{0\}$ ) satisfying $\int_{\mathbb{R}}\left(1 \wedge x^{2}\right) v(d x)<\infty$. We only consider nondeterministic processes $X$, which is equivalent to saying that $\sigma^{2}+v(\mathbb{R} \backslash\{0\})>0$. We say that: $X$ is centered if $\mathbb{E} X_{t}=0$ for all $t$; spectrally positive if $v(-\infty, 0)=0$; spectrally negative if $v(0, \infty)=0$. If $X_{1}$ has a stable distribution with index $\alpha \in(0,2]$ then we say that $X$ is an $\alpha$-stable Lévy process and denote it by $\mathcal{L}^{(\alpha)}$. For more background on Lévy processes we refer the reader to [2] and references therein.

For an interval $B \subseteq[0, \infty)$, we denote by $D(B)$ the space of real-valued càdlàg functions on $B$ equipped with the usual Skorokhod $J_{1}$ topology; see, for example, [11, Chap. VI].

In the sequel we will encounter the Mittag-Leffler distribution; see, for example, [4, p. 329]. A positive random variable $\mathcal{M} \mathcal{L}_{\alpha}$ is said to have a Mittag-Leffler distribution with parameter $\alpha \in(0,1]$ if its Laplace-Stieltjes transform (LST) is given by

$$
\mathbb{E} \exp \left(-s \mathcal{M} \mathcal{L}_{\alpha}\right)=\frac{1}{1+s^{\alpha}}
$$

Observe that $\mathcal{M} \mathcal{L}_{1}$ has the 1-exponential distribution.

We will also make use of some standard notation. For two functions $f, g$ we shall write $f(x) \sim g(x)$ as $x \rightarrow x_{0} \in[0, \infty]$ to mean $\lim _{x \rightarrow x_{0}} f(x) / g(x)=1$. The class of regularly varying functions with index $\alpha$ shall be denoted by $\mathcal{R} \mathcal{V}_{\alpha}$.

In what follows we shall also write

$$
\bar{X}^{(a)}=\sup _{t \geq 0} X_{t}^{(a)}, \quad \bar{S}^{(a)}=\max _{n \geq 0} S_{n}^{(a)},
$$

where, for each $a>0,\left\{X_{t}^{(a)}: t \geq 0\right\}$ is a Lévy process and $S_{n}^{(a)}=\sum_{k=1}^{n} Y_{k}^{(a)}$ is the $n$th partial sum of a sequence of random variables $\left\{Y_{n}^{(a)}: n \geq 1\right\}$ with $S_{0}^{(a)}=0$. 


\section{Main theorem}

Theorem 1 For each $a>0$, let $\left\{Y_{n}^{(a)}: n \geq 1\right\}$ be a sequence of i.i.d. random variables and $\left\{X_{t}^{(a)}: t \geq 0\right\}$ be a Lévy process. Assume that for each $a, Y_{1}^{(a)} \stackrel{d}{=} X_{1}^{(a)}$, $\mu^{(a)}=\mathbb{E} Y_{1}^{(a)}<0$ and $\mu^{(a)} \uparrow 0$ as $a \downarrow 0$. Then, for some random variable $\mathcal{R}$,

$$
\Delta(a) \max _{n \geq 0} S_{n}^{(a)} \stackrel{d}{\rightarrow} \mathcal{R} \Longleftrightarrow \Delta(a) \sup _{t \geq 0} X_{t}^{(a)} \stackrel{d}{\rightarrow} \mathcal{R}, \quad \text { as } a \downarrow 0,
$$

where $\Delta(\cdot)$ is a normalizing function such that $\Delta(a) X_{1}^{(a)} \rightarrow 0$ almost surely.

Remark 1 The assumptions of the above theorem are natural:

- $\mu^{(a)} \uparrow 0$ assures that, for each $a, \bar{X}^{(a)}$ and $\bar{S}^{(a)}$ are finite random variables. Moreover, $\bar{X}^{(a)}$ and $\bar{S}^{(a)}$ tend to infinity with $a$. Therefore the function $\Delta(\cdot)$ tends to zero and can be seen as the speed of convergence in (1).

- $\Delta(a) X_{1}^{(a)} \rightarrow 0$ is satisfied in all typical applications; for instance, when $X_{t}^{(a)}=$ $X_{t}-a t$ for a fixed Lévy process $X$, see Sects. 4.1 and 4.2; or there exists a function $d(a) \rightarrow \infty$ such that $X_{d(a) t}^{(a)} \rightarrow X$ in $D[0, \infty)$, see Sect. 4.3.

The distribution of the random variable $\mathcal{R}$ can be computed in several cases; we will get back to it in Remark 4 .

Proof of Theorem 1 With $\bar{R}(x):=1-R(x)$, where $R$ is the distribution function of $\mathcal{R}$, it is enough to show that, as $a \downarrow 0$,

$$
\mathbb{P}\left(\Delta(a) \bar{S}^{(a)}>x\right) \rightarrow \bar{R}(x) \quad \Longleftrightarrow \mathbb{P}\left(\Delta(a) \bar{X}^{(a)}>x\right) \rightarrow \bar{R}(x),
$$

for any continuity point $x$ of $R$.

Assume that $\Delta(a) \bar{S}^{(a)} \stackrel{d}{\rightarrow} \mathcal{R}$, the converse implication follows in the same manner.

Observe that $\bar{S}^{(a)} \stackrel{d}{=} \max _{n \geq 0} X_{n}^{(a)}$. Thus, we trivially have

$$
\mathbb{P}\left(\Delta(a) \bar{X}^{(a)}>x\right) \geq \mathbb{P}\left(\Delta(a) \bar{S}^{(a)}>x\right) .
$$

On the other hand, for any $x_{0}>0$,

$$
\begin{aligned}
\mathbb{P}\left(\Delta(a) \bar{X}^{(a)}>x\right) \leq & \mathbb{P}\left(\Delta(a) \max _{n \geq 0} X_{n}^{(a)}>x-x_{0}\right) \\
& +\mathbb{P}\left(\Delta(a) \bar{X}^{(a)}>x, \Delta(a) \max _{n \geq 0} X_{n}^{(a)} \leq x-x_{0}\right) \\
= & \mathbb{P}\left(\Delta(a) \bar{S}^{(a)}>x-x_{0}\right) \\
& +\mathbb{P}\left(\Delta(a) \bar{X}^{(a)}>x, \Delta(a) \max _{n \geq 0} X_{n}^{(a)} \leq x-x_{0}\right) .
\end{aligned}
$$

We use an argument similar to the one in [17, 25]. Define $\tau^{(a)}(x)=\inf \{t \geq 0$ : $\left.\Delta(a) X_{t}^{(a)}>x\right\}$, so that $\tau^{(a)}$ is a stopping time [2, Corollary 8]. Now the second 
term on the right-hand side of the above inequality can be bounded from above by

$$
\begin{aligned}
& \mathbb{P}\left(\tau^{(a)}(x)<\infty, \Delta(a)\left(\inf _{t \in\left[\tau^{(a)}(x), \tau^{(a)}(x)+1\right]}\left(X_{t}^{(a)}-X_{\tau^{(a)}(x)}^{(a)}\right)\right) \leq-x_{0}\right) \\
& \quad=\mathbb{P}\left(\tau^{(a)}(x)<\infty\right) \mathbb{P}\left(\Delta(a) \inf _{t \in[0,1]} X_{t}^{(a)} \leq-x_{0}\right),
\end{aligned}
$$

where we used the strong Markov property in the last equality. Thus,

$$
\mathbb{P}\left(\Delta(a) \bar{X}^{(a)}>x\right) \mathbb{P}\left(\Delta(a) \inf _{t \in[0,1]} X_{t}^{(a)}>-x_{0}\right) \leq \mathbb{P}\left(\Delta(a) \bar{S}^{(a)}>x-x_{0}\right) .
$$

Now $\Delta(a) X_{1}^{(a)} \rightarrow 0$ a.s. implies that the finite-dimensional distributions of $\left\{\Delta(a) X_{t}^{(a)}: t \in[0,1]\right\}$ converge to zero a.s. and thus by [22] the whole process converges to 0 in $D[0,1]$. Applying the continuous mapping theorem with the infimum (over [0,1]) map yields $\Delta(a) \inf _{t \in[0,1]} X_{t}^{(a)} \rightarrow 0$. Thus, combining formulas (2) and (3) we get

$$
\bar{R}(x) \leq \liminf _{a \rightarrow 0} \mathbb{P}\left(\Delta(a) \bar{X}^{(a)}>x\right) \leq \limsup _{a \rightarrow 0} \mathbb{P}\left(\Delta(a) \bar{X}^{(a)}>x\right) \leq \bar{R}\left(x-x_{0}\right) .
$$

The result follows by letting $x_{0} \rightarrow 0$.

Remark 2 In the following section, we shall use the if part of Theorem 1 to derive various limit theorems for suprema of Lévy processes. It is worth noting however that the only if part could also be used to derive limit theorems for suprema of random walks. A variation of this approach has been undertaken in Szczotka and Woyczyński [23], where first a heavy-traffic limit theorem is derived in continuous time and then this theorem is used to claim an analogous behavior in discrete time.

\section{Special instances}

Theorem 1 provides a tool for translating limit theorems for random walks to their analogues in the Lévy setting. In this section we shall focus our attention on some seminal results about the convergence of the maxima of random walks and reformulate them to the Lévy case. We illustrate each special case that we consider with a remark that explains an alternative way of obtaining the particular result via a direct approach undertaken in the literature. These remarks, albeit short, are rigorous enough to act as alternative proofs. Let us start with the case in which the underlying processes are spectrally positive, which is closely related to the queueing setting via the compound Poisson process.

\subsection{Spectrally positive processes}

For a sequence of zero mean, i.i.d. random variables $\left\{Y, Y_{n}: n \geq 1\right\}$, the question of how fast $\bar{S}^{(a)}=\max _{n \geq 0}\left(S_{n}-n a\right)$ grows as $a \downarrow 0$ was first posed by Kingman $[13,14]$. Kingman in his proof assumed exponential moments of $|Y|$ and used 
Wiener-Hopf factorization to obtain the Laplace transform of $\bar{S}^{(a)}$. Prokhorov [18] generalized Kingman's result to the case when only the second moment of $Y$ is finite. His approach was based on the functional Central Limit Theorem. These two approaches have become classical and have both been used to prove various heavytraffic results. The analytical approach of Kingman was used by Boxma and Cohen [5] (see also Cohen [6]) to study the limiting behavior of $\bar{S}^{(a)}$ in the case of infinite variance. They proved that if $\mathbb{P}(Y>x)$ is regularly varying at infinity with a parameter $\alpha \in(1,2)$ (and under some additional assumptions), then there exists a function $\Delta(\cdot)$ such that $\Delta(a) \bar{S}^{(a)}$ converges in law to a proper random variable.

Theorem 5.1 from Boxma and Cohen [5] acts as the first application of our main result. For a Lévy measure $v$ define

$$
r(s):=\int_{0}^{\infty}\left(e^{-s x}-1+s x\right) v(d x) .
$$

For a Lévy process $X \equiv\left\{X_{t}: t \geq 0\right\}$, let $F$ be the distribution function of $X_{1}$ and set $\bar{F}:=1-F$. With $\alpha>0$, Theorem 8.2.1 from [4] asserts that $\bar{F} \in \mathcal{R \mathcal { V } _ { - \alpha }}$ if and only if $v(x, \infty) \in \mathcal{R} \mathcal{V}_{-\alpha}$, where $v$ is the Lévy measure of $X$; moreover: $\bar{F}(x) \sim v(x, \infty)$, as $x \rightarrow \infty$. This, combined with Theorem 5.1 from [5] and Theorem 1, yields:

Proposition 1 Let $X$ be a spectrally positive Lévy process such that $v(x, \infty) \in$

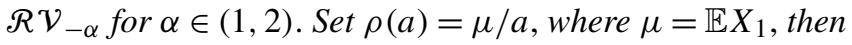

$$
\Delta(\rho(a)) \sup _{t \geq 0}\left(X_{t}-a t\right) \stackrel{d}{\rightarrow} \mathcal{M} \mathcal{L}_{\alpha-1}, \quad \text { as } \rho(a) \uparrow 1,
$$

where $\Delta(x)=d(x) / \mu$ and $d(x)$ is such that

$$
r(d(x)) \sim d(x) \frac{1-x}{x} \mu^{\alpha}, \quad \text { as } x \uparrow 1 .
$$

See also [5, 20] for possible refinements of the assumption on regular variation in this special case.

Remark 3 It is possible to prove Proposition 1 using a direct approach like the one in [5]. Let $X_{t}^{(a)}=X_{t}-a t, a>\mu$, then the Pollaczek-Khinchine formula (see, for example, [1] Chap. IX) yields

$$
\mathbb{E} e^{-\lambda \bar{X}^{(a)}}=\frac{\lambda \varphi_{a}^{\prime}(0)}{\varphi_{a}(\lambda)}<\infty, \quad \text { for } \lambda>0,
$$

where $\varphi_{a}(\lambda)=\log \mathbb{E} \exp \left(-\lambda\left(X_{1}-a\right)\right)$. Substituting $\varphi_{a}(\lambda)=\lambda \varphi_{a}^{\prime}(0)+r(\lambda)$ yields

$$
\frac{\lambda \varphi_{a}^{\prime}(0)}{\varphi_{a}(\lambda)}=\frac{1}{1+\frac{r(\lambda)}{\lambda \varphi_{a}^{\prime}(0)}},
$$

where we assumed $\sigma=0$ for simplicity. Let $\lambda=s \Delta(\rho(a))$ with $\Delta(\cdot)$ as in Proposition 1. Using Theorem 8.1.6 from [4] one infers that, under the assumption $v(x, \infty) \in$ 
$\mathcal{R} \mathcal{V}_{-\alpha}, r$ is a regularly varying function at 0 with index $\alpha$. We necessarily have $d(x) \downarrow 0$, as $x \uparrow 1$. Hence, as $\rho(a) \uparrow 1$,

$$
\frac{r(\lambda)}{\lambda \varphi_{a}^{\prime}(0)} \sim\left(\frac{s}{\mu}\right)^{\alpha-1} \frac{r(d(\rho(a)))}{d(\rho(a))(a-\mu)}=\frac{s^{\alpha-1}}{\mu^{\alpha}} \frac{r(d(\rho(a)))}{d(\rho(a))} \frac{\rho(a)}{1-\rho(a)} \sim s^{\alpha-1} .
$$

\subsection{Asymptotically stable processes}

Proposition 1 limits the class of Lévy processes under consideration to spectrally positive. Further improvements of the result from [5] by Furrer [10] and Resnick and Samorodnitsky [20] assumed that the random walk belongs to the domain of attraction of a spectrally positive stable law and relied on functional limit theorems. Shneer and Wachtel [21] relaxed this assumption to allow the random walk to belong to the domain of attraction of any stable law. The main result from [21] acts as the second instance of an application of Theorem 1.

Proposition 2 Let $X$ be a centered Lévy process such that the random variable $X_{1}$ belongs to the domain of attraction of a stable law $\mathcal{L}_{1}^{(\alpha)}$ with index $\alpha \in(1,2]$. That is, there exists a sequence $\{d(n): n \geq 0\}$ such that

$$
\frac{X_{n}}{d(n)} \stackrel{d}{\rightarrow} \mathcal{L}_{1}^{(\alpha)}, \quad \text { as } n \rightarrow \infty
$$

Then,

$$
\Delta(a) \sup _{t \geq 0}\left(X_{t}-a t\right) \stackrel{d}{\rightarrow} \sup _{t \geq 0}\left(\mathcal{L}_{t}^{(\alpha)}-t\right), \quad \text { as } a \downarrow 0, \quad \text { where } \Delta(a)=\frac{1}{d(n(a))}
$$

and $n(a)$ is such that

$$
\operatorname{an}(a) \sim d(n(a)), \quad \text { as } a \downarrow 0 .
$$

Remark 4 It is well known that the sequence $d(n)$ in Proposition 2 is regularly varying with index $1 / \alpha$. Therefore, Proposition 2 implies that, with $X_{t}^{(a)}=X_{t}-a t, \bar{X}^{(a)}$ grows as a regularly varying function (at zero) with index $-1 /(\alpha-1)$. If $\mathcal{L}^{(\alpha)}$ is spectrally negative, then the distribution of $\mathcal{R}=\sup _{t \geq 0}\left(\mathcal{L}_{t}^{(\alpha)}-t\right)$ is exponential; see, for example, [3, Proposition 5]. If $\mathcal{L}^{(\alpha)}$ is spectrally positive, then, as seen in Proposition 1, the limiting random variable has a Mittag-Leffler distribution; see, for example, [12, Theorem 4.2]. If $\mathcal{L}^{(\alpha)}$ is symmetric, then one can give the LaplaceStieltjes transform of $\mathcal{R}$; see [23, Theorem 8]. In the other cases it might be infeasible to compute the explicit form of the distribution; however, one can easily find its tail asymptotics $\mathbb{P}(\mathcal{R}>x) \sim C x^{1-\alpha}$. For more details on the supremum distribution of a Lévy process, see [23].

Remark 5 Shneer and Wachtel [21] showed that both classical approaches, i.e., via Wiener-Hopf factorization and via a functional central limit theorem, can be applied to obtain their result. Moreover, the technical difficulties arising from these methods 
can be overcome using a generalization of Kolmogorov's inequality based on a result by Pruitt [19]. A similar result is also available for Lévy processes and can also be found in [19]. Let us introduce $V(x)=\int_{|y| \leq x} y^{2} v(d y)$, the truncated second moment of the Lévy measure $v$. Under the assumptions of Proposition $2, V \in \mathcal{R} \mathcal{V}_{2-\alpha}$. Moreover, Pruitt [19, Sect. 3] asserts that there exists a constant $C$ such that

$$
\mathbb{P}\left(\sup _{s \leq t} X_{s} \geq x\right) \leq C \frac{t V(x)}{x^{2}} .
$$

Using the regular variation of $V,(6)$ and (7), for any fixed $T>0$ there exist constants $C_{1}, C_{2}>0$ such that

$$
\begin{aligned}
\mathbb{P}\left(\sup _{t \geq n(a) T}\left(X_{t}-a t\right) \geq 0\right) & \leq \sum_{k=0}^{\infty} \mathbb{P}\left(\sup _{t \leq 2^{k+1} n(a) T} X_{t} \geq 2^{k} a n(a) T\right) \\
& \leq C_{1} \frac{V(a n(a) T)}{a^{2} n(a) T} \sum_{k=0}^{\infty}\left(2^{k}\right)^{1-\alpha} \leq C_{2} \frac{V(d(n(a)))}{c^{2}(n(a))} n(a) T^{1-\alpha}
\end{aligned}
$$

The sequence $d(n)$ can be defined as $d(n):=\inf \left\{t>0: V(t) \leq t^{2} / n\right\}$, therefore the last expression tends to zero, uniformly in $a>0$, as $T$ tends to infinity. This, combined with the classical functional limit theorem corresponding to (5) and the fact that for a fixed $T>0$, supremum on $[0, T]$ is a continuous map, yields the result of Proposition 2.

On the other hand, as a consequence of the Wiener-Hopf factorization (see [16, Chap. 6]), with $X_{t}^{(a)}=X_{t}-a t$, the LST of $\bar{X}^{(a)}$ is given by

$$
\mathbb{E} e^{-\lambda \bar{X}^{(a)}}=\exp \left(-\int_{0}^{\infty} \frac{1}{t} \mathbb{E}\left(1-e^{-\lambda\left(X_{n(a) t}-a n(a) t\right)}, X_{n(a) t}-a n(a) t>0\right) d t\right) .
$$

Plugging in $\lambda=\Delta(a) s$ for $s>0$, from (5) and (6) it follows that, as $a \downarrow 0$, this expression tends to

$$
\mathbb{E} e^{-s \mathcal{R}}=\exp \left(-\int_{0}^{\infty} \frac{1}{t} \mathbb{E}\left(1-e^{-s\left(\mathcal{L}_{t}^{(\alpha)}-t\right)}, \mathcal{L}_{t}^{(\alpha)}-t>0\right) d t\right),
$$

the LST of $\mathcal{R}=\sup _{t \geq 0}\left(\mathcal{L}_{t}^{(\alpha)}-t\right)$, provided that we can interchange the limit with the integral. This follows by using the dominated convergence theorem. For big values of $t$, say $t>T$ and some $C_{3}, C_{4}>0$, we can estimate the integrand by (cf. (7) and (8))

$$
\frac{1}{t} \mathbb{P}\left(X_{n(a) t}>a n(a) t\right) \leq C \frac{V(a n(a) t)}{a^{2} n(a) t^{2}} \leq C_{3} t^{-\alpha} \frac{V(d(n(a)))}{c^{2}(n(a))} n(a) \leq C_{4} t^{-\alpha} .
$$

For $t \leq T$ and some $C_{5}>0$, one can simply bound the integrand by (cf. (7))

$$
C_{5} s t^{1 / \alpha-1} \mathbb{E}\left(\mathcal{L}_{1}^{(\alpha)}, \mathcal{L}_{1}^{(\alpha)}>0\right) .
$$




\subsection{Heavy-traffic invariance principle}

A general principle called the heavy-traffic invariance principle has been established by Szczotka and Woyczyński [23]; see also [7, 8, 24]. This principle asserts under what condition one can infer the limiting distributions of maxima of random walks from functional limit theorems. According to Theorem 1 this principle can be also reformulated to the Lévy setting. Therefore we conclude the paper with the following theorem:

Proposition 3 (Heavy-Traffic Invariance Principle) For a family of Lévy processes $\left\{X_{t}^{(a)}: t \geq 0\right\}$ define $\mu^{(a)}=\mathbb{E} X_{1}^{(a)}<0$ and assume that $\mu^{(a)} \uparrow 0$. Moreover, assume that there exist functions $d(\cdot)$ and $\Delta(\cdot)$, such that the following conditions hold:

(I) $d(a) \Delta(a)\left|\mu^{(a)}\right| \rightarrow \beta \in(0, \infty)$.

(II) $\Delta(a)\left\{X_{d(a) t}^{(a)}-t d(a) \mu^{(a)}: t \geq 0\right\} \stackrel{d}{\rightarrow}\left\{X_{t}: t \geq 0\right\}$ in $D[0, \infty)$, where $X$ is a Lévy process.

(III) the family $\left\{\Delta(a) \bar{X}^{(a)}: a>0\right\}$ is tight.

Then,

$$
\Delta(a) \sup _{t \geq 0} X_{t}^{(a)} \stackrel{d}{\rightarrow} \sup _{t \geq 0}\left(X_{t}-\beta t\right)
$$

See Theorem 2 from Szczotka and Woyczyński [23] for an extension to processes $X^{(a)}$ with stationary increments in the case $X$ is stochastically continuous.

Open Access This article is distributed under the terms of the Creative Commons Attribution Noncommercial License which permits any noncommercial use, distribution, and reproduction in any medium, provided the original author(s) and source are credited.

\section{References}

1. Asmussen, S.: Applied Probability and Queues. Springer, Berlin (2003)

2. Bertoin, J.: Lévy Processes. Cambridge University Press, Cambridge (1990)

3. Bingham, N.H.: Fluctuation theory in continuous time. Adv. Appl. Probab. 7, 705-766 (1975)

4. Bingham, N.H., Goldie, C.M., Teugels, J.L.: Regular Variation. Cambridge University Press, Cambridge (1987)

5. Boxma, O.J., Cohen, J.W.: Heavy-traffic analysis for the $G I / G / 1$ queue with heavy-tailed distributions. Queueing Syst. 33, 177-204 (1999)

6. Cohen, J.W.: Random walk with a heavy-tailed jump distribution. Queueing Syst. 40, 35-73 (2002)

7. Czystołowski, M., Szczotka, T.: Tightness of stationary waiting times in Heavy Traffic for GI/GI/1 queues with thick tails. Probab. Math. Stat. 27, 109-123 (2007)

8. Czystołowski, M., Szczotka, T.: Queueing approximation of suprema of spectrally positive Lévy process. Queueing Syst. 64, 305-323 (2010)

9. Doney, R.A.: Stochastic bounds for Lévy processes. Ann. Probab. 32, 1545-1552 (2004)

10. Furrer, H.: Risk theory and heavy-tailed Lévy processes. Ph.D. thesis, ETH, Zurich (1997)

11. Jacod, J., Shiryaev, A.N.: Limit Theorems for Stochastic Processes. Springer, Berlin (1987)

12. Kella, O., Whitt, W.: Queues with server vacations and Lévy processes with secondary jump input. Ann. Appl. Probab. 1, 104-117 (1991)

13. Kingman, J.F.C.: The single server queue in heavy traffic. Proc. Camb. Philol. Soc. 57, 902-904 (1961) 
14. Kingman, J.F.C.: On queues in heavy traffic. J. R. Stat. Soc., Ser. B, Stat. Methodol. 24, 383-392 (1962)

15. Kingman, J.F.C.: The heavy traffic approximation in the theory of queues. In: Smith, W.L., Wilkinson, W.E. (eds.) Proceedings of Symposium on Congestion Theory, pp. 137-159. University of North Carolina Press, Chapel Hill (1965)

16. Kyprianou, A.E.: Introductory Lectures on Fluctuations of Lévy Processes with Applications. Springer, Berlin (2006)

17. Maulik, K., Zwart, B.: Tail asymptotics for exponential functionals of Lévy processes. Stoch. Process. Appl. 116, 156-177 (2006)

18. Prokhorov, Ju.V., Transition phenomena in queueing processes. I. Liet. Mat. Rink. 9, 199-205 (1963)

19. Pruitt, W.E.: The growth of random walks and Lévy processes. Ann. Probab. 9, 948-956 (1981)

20. Resnick, S., Samorodnitsky, G.: A heavy traffic limit theorem for workload processes with heavy tailed service requirements. Manag. Sci. 46, 1236-1248 (2000)

21. Shneer, S., Wachtel, V.: Heavy-traffic analysis of the maximum of an asymptotically stable random walk. Technical Report 2009-005, EURANDOM (2009)

22. Skorokhod, A.V.: Limit theorems for stochastic processes with independent increments. Theory Probab. Appl. 11, 138-171 (1957)

23. Szczotka, T., Woyczyński, W.A.: Distributions of suprema of Lévy processes via heavy traffic invariance principle. Probab. Math. Stat. 23, 251-272 (2003)

24. Szczotka, T., Woyczyński, W.A.: Heavy-tailed dependent queues in heavy traffic. Probab. Math. Stat. 24, 67-96 (2004)

25. Willekens, E.: On the supremum of an infinitely divisible process. Stoch. Process. Appl. 26, 173-175 (1987) 